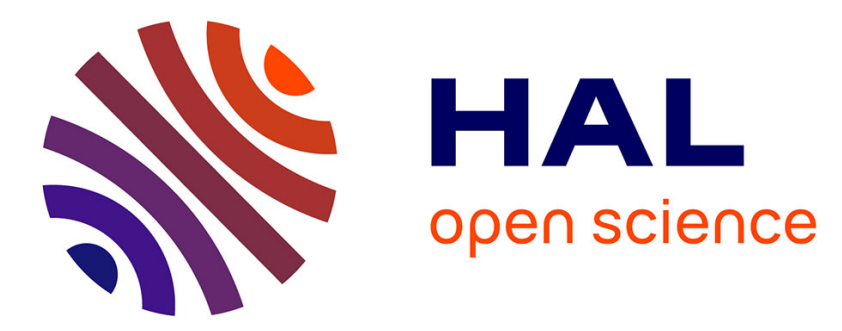

\title{
The Influence of Defects Localization and Stable Mezoparticles Formation on the Behaviour of Material under Impact Loading \\ E. Prockuratova, D. Indejtchev
}

\section{- To cite this version:}

E. Prockuratova, D. Indejtchev. The Influence of Defects Localization and Stable Mezoparticles Formation on the Behaviour of Material under Impact Loading. Journal de Physique IV Proceedings, 1997, 07 (C3), pp.C3-815-C3-820. 10.1051/jp4:19973138 . jpa-00255425

HAL Id: jpa-00255425

https://hal.science/jpa-00255425

Submitted on 1 Jan 1997

HAL is a multi-disciplinary open access archive for the deposit and dissemination of scientific research documents, whether they are published or not. The documents may come from teaching and research institutions in France or abroad, or from public or private research centers.
L'archive ouverte pluridisciplinaire HAL, est destinée au dépôt et à la diffusion de documents scientifiques de niveau recherche, publiés ou non, émanant des établissements d'enseignement et de recherche français ou étrangers, des laboratoires publics ou privés. 


\title{
The Influence of Defects Localization and Stable Mezoparticles Formation on the Behaviour of Material under Impact Loading
}

\author{
E.I. Prockuratova and D.A. Indejtchev
}

Russia Academy of Science, Institute of Mechanical Engineering Problems, V.O. Bolchoi 61, 199178 St. Petersbourg, Russia

\begin{abstract}
The paper is devoted to the investigation of behaviour of material under impact loading. Two structure levels of deformation was took into account. At microlevel the conditions of existence of stable dislocation groups formation and their distribution were obtained. These groups were considered as an inclutions with finite mass at the next structure level of deformation. The influence of the mass and position of the inclusion on the stress field in material was studied. For some particular forms of loading it was shown how deformation depends on the characteristics on the inclusion.
\end{abstract}

\section{MODELLING OF THE PROCESSES OF DYNAMIC LOCALIZATION ON MICRO- AND MEZOLEVEL OF DEFORMATION}

Nowadays investigations of behaviour of material under impact loading develop in two directions. Both directions are based on the experimentally established fact of hierarchy of structural levels of deformation. $[1,2]$

At microlevel of deformation the dynamics and self-organization of defects were investigated on the basis of the dislocation theory equations. However examination of stress-strain state of medium as a whole is beyond the dislocation approach possibilities.

Behaviour of material at mezolevel is conditioned by the wave process and by the equations of solids mechanics. In addition, collective effects of dislocation motion lead to the rise and movement of particles at the next structural level(mezoparticles).

So, to carry out the transition from microlevel to macrolevel of deformation we have to study the conditions of formation of mezoparticles as stable dislocation configurations and to take into account their influence of the process of wave propagation in the dynamically loaded material.

\subsection{The conditions of the dislocation distribution localization under dynamic loading.}

It was shown preliminarily that as the relaxation time of dislocation is far less then time of macroprocess, then if we solve the problem of dynamic behaviour of material with dislocation structure we can consider the evolution of dislocations independently. When we regard this problem we deal with the equation of dislocation evolution in the form

$$
\frac{\partial \alpha}{\partial t}+\frac{\partial(\alpha u)}{\partial x}=J(\alpha, u)
$$

where $\alpha, u$ are the density and velocity of dislocation and $t$ is the time of microprocess. The equation was investigated for two particular cases of right hand side form. Thus in [3] we considered the case when

$$
J=a \alpha-\beta \alpha^{2}
$$


i.e $J$ depend on dislocation density only. It was shown that the solution of (1.1) will be oscillating with period $T=2 \pi \alpha_{0} / \nu$ when applied stress $S_{0}$ is large comparing with some critical

$$
S_{c r}=\sqrt{\frac{8 D R}{k}\left(a \alpha_{0}-\beta \alpha_{0}^{2}\right)}
$$

and

$$
\nu=\sqrt{\frac{a \alpha_{0}-\beta \alpha_{0}^{2}}{2 \pi R k}+\frac{S_{0}^{2}}{16 D^{2} R^{2}}}
$$

If right side hand of equation for evolution of dislocation structure is in the following form[4]

$$
J(\alpha, u)=a \alpha u-\beta(\alpha u)^{2}
$$

i.e depend only on dislocation flow, then dislocation distribution is localize near some point $x_{*}$ and has the form

$$
\alpha=\phi_{0}+\phi_{2}\left(x-x_{*}\right)^{2}
$$

As point $x_{*}$ is an extreme point of the dislocation density a region of localization of dislocation distribution must exist in the vicinity of this point. To find $x_{*}$ and parameters of dislocation distribution near the point stress and dislocation flow in (1.1) were represent as a series in terms of powers of $\left(x-x_{*}\right)$ up to the second order. Here we took into account that we can consider $u$ as $[3,4]$

$$
u=k\left[\sigma(x)-D \int_{-\infty}^{+\infty} \frac{\alpha(\xi, t)}{\xi-x} d \xi\right]
$$

Then we derive an equation

$$
\mathcal{A}+\mathcal{B}\left(x-x_{*}\right)+\mathcal{C}\left(x-x_{*}\right)^{2}=0
$$

As (1.8) is valid for any $x$ in the vicinity of $x_{*}$ then

$$
\mathcal{A}=\mathcal{B}=\mathcal{C}=0
$$

where $\mathcal{A}, \mathcal{B}, \mathcal{C}$ are the functions of $x_{*}, \phi_{0}, \phi$. Here we have supposed that external stress changes according to $\sigma=\sigma_{0}+\sigma_{1} \exp (-\delta x)$ Expression (1.9) is a closed system of three algebraic equations with three unknowns $\phi_{0}, \phi_{2}$ and $x_{*}$. In [4] this system was solved in two particular cases, both the case of a high stress gradient and a low one, i.e. $\delta x_{*} \gg a x_{*}$ and $\delta x_{*} \ll a x_{*}$. In the first case the solution of the system is

$$
\begin{aligned}
& \phi_{0}=\frac{a}{4 k \beta \sigma_{0}}\left[\sqrt{\frac{4 k \beta \sigma_{0}^{2}}{D R a^{2}}+1}+1\right] \\
& \phi_{2}=\frac{a \sigma_{0}}{16 D R}\left[\sqrt{\frac{4 k \beta \sigma_{0}^{2}}{D R a^{2}}+1}-3\right]
\end{aligned}
$$

As $x_{*}$ is an extremal point of function $\alpha(x), \phi_{2}$ must be negative and so the following inequality must be valid

$$
\sigma_{0}^{2}<\frac{2 D R}{k} \cdot \frac{a^{2}}{\beta}
$$

In the second case the solution of the problem has the form

$$
\begin{gathered}
\phi_{0}=\sqrt{\frac{a}{2 D R k \beta \delta}} \\
\phi_{2}=\frac{1}{2}\left[\delta^{2} \sqrt{\frac{a}{2 D R k \beta \delta}}-\frac{\sigma_{0} \delta}{2 D R}\right]
\end{gathered}
$$


and localization condition is

$$
\sigma_{0}^{2}<\sqrt{\frac{a \delta 2 D R}{k \beta}}
$$

These two particular cases show that dislocation structure localization is possible under certain conditions,(1.12) and (1.15). Moreover if in the second case we must take into account both the loading stress parameters $\delta$ and coefficients of dislocation structure ( $a$ and $\beta$ ), in the first case the coefficients of dislocation structure are most important for dislocation group formation.

\subsection{Connection between micro- and mezolevel of deformation.}

In twolevel description the evolution of micro- and mezoparticles is based on the following system of evolution equations [5]

$$
\begin{gathered}
\frac{\partial \alpha_{1}}{\partial t}+\frac{\partial}{\partial x}\left(\alpha_{1} V_{1}\right)=a_{1} \alpha_{1}-\beta_{1} \alpha_{1}^{2}+k_{1} \alpha_{1} \alpha_{2} \\
\frac{\partial \alpha_{2}}{\partial t}+\frac{\partial}{\partial x}\left(\alpha_{2} V_{2}\right)=\beta_{2} \alpha_{2}-k_{1} \alpha_{1} \alpha_{2}
\end{gathered}
$$

Here $V_{1}, V_{2}$ are average dislocation and mezoparticle velocity and $\alpha_{1}, \alpha_{2}$ are their density correspondingly. $a_{1}, \beta_{1}, \beta_{2}, k$ are the constants of material and of the conditions of loading. The solution of the system permits us to investigate the evolution of micro- and mezostructure and their interaction.

In the case of dynamic loading the velocity of dislocations $V_{1}$ describes by equation (1.7). The velocity of mezoparticles was found in following assumption.

a.Mezoparticle is superdislocation.

b.Potential energy of mezoparticle is the following

$$
U(x, t)=\Omega\left[\sigma-D_{1} \int_{0}^{R_{2}} \frac{\alpha_{2}(\xi, t)}{\xi-x} d \xi\right]^{2}
$$

c.The initial distribution at mezolevel in phase space is described by Maxwell-Boltsman function

$$
f\left(V_{2}, x, t\right)=\frac{1}{(2 \pi \theta)^{1 / 2}} \exp \left[-\frac{\left(V_{2}-V_{c r}\right)^{2}}{2 \theta}-\frac{U(x, t)}{\theta}\right]
$$

the latter allows us to determine the average velocity of mezoparticles.

The problem was solved by successive approximations method. As a zero approximation the solution of the form

$$
\alpha_{1}=\alpha_{10}+\Psi, \alpha_{2}=\alpha_{20}+W
$$

is being search for the system (1.16). Here $\alpha_{10}, \alpha_{20}$ are the initial distribution density and $\Psi, W$ are the small fluctuations of the density. As a zero approximation at microlevel we can chose one of the known distributions obtained previously. For example, it was found in [4] for stationary case

$$
\Psi(x)=\frac{S}{4 D_{1} R_{1}} x+\alpha_{10} \ln |\cos (\nu x)|
$$

Then zero approximation at mezolevel which takes into account zero approximation at microlevel is

$$
W(x)=l_{1}\left[f(x) G(c)-l_{2} \frac{\partial f(x)}{\partial x} \frac{\partial G(c)}{\partial x}\right]-l_{1}\left[f(c) G(x)-l_{2} \frac{\partial f(c)}{\partial x} \frac{\partial G(x)}{\partial x}\right]+\mathcal{E} i(x)
$$

where 


$$
\begin{array}{r}
f(x)=m_{3}\left[k_{1} \Psi(x)-\phi\right] \\
G(x)=\exp \left[-m_{1}(c-x) / 2\right] \sin \left[\nu_{2}(c-x) / 2+\Phi\right]
\end{array}
$$

and $m_{1}, m_{2}, m_{3}, l_{1}, l_{2}, \Phi, \phi$ are constants depending on parameters of material and onf the loading conditions. Constant $c$ is determined by the boundary conditions, i.e. by the amplitude and velocity of loading.

Then we have found the first approximation to the dislocation density. Here we take into account that while the mezolevel being joined the contribution of the microlevel into the whole deformation must decrease [7]. And beginning from some moment the next structural level is to be involved. So we can find $\alpha_{1}$ in following form

$$
\alpha_{1}=\alpha_{10}+\Psi(x)+\Psi_{1}
$$

The problem was solved by Laplace transformation method and it has following solution

$$
\Psi_{1}=\frac{\alpha_{10} \alpha_{20}}{p_{1}-p_{2}}\left[\frac{1}{p_{2}}-\frac{1}{p_{1}}+\frac{1}{p_{1}} \exp \left(p_{1} x\right)-\frac{1}{p_{2}} \exp \left(p_{2} x\right)\right]+\alpha_{20}\left(\Psi * \Psi_{11}+\alpha_{10}\left(W * \Psi_{11}\right)\right.
$$

where $\Psi_{11}=\frac{1}{p_{1}-p_{2}}\left[\exp \left(p_{1} x\right)-\exp \left(p_{2} x\right)\right]$ and symbol $*$ means mathematical operation of folding $p_{1}$ and $p_{2}$ are the roots of equation

$$
p^{2}+n_{1} p+n_{2}=0
$$

$n_{1}, n_{2}$ depend on parameters of material and conditions of loading.

As was mentioned before the role of every level of deformation is decreased when the next structural level enjoined in processes of deformation.

So, if we want to describe deformation at mezolevel we must take into account dislocation processes exclusively.But if we want to describe macrodeformation we have to consider processes at mezolevel of deformation and can neglect processes taking place at dislocation level.

\section{THE INFLUENCE OF MEZOPARTICLES ON THE WAVE PROPAGATION IN DYNAMIC LOADING MATERIAL}

In this section we will consider the problem of the wave propagation in one-dimensional beam of finite length $L$ with an inclusion in point $l$.

We can regard mezoparticle which has formed as a result of dislocation structure localization as an inclusion. Let's assume that such an inclusion has its own mass $M$ and that its size is negligible with respect to the length of beam. We have not take into account stiffness of the inclusion and thickness of the beam. Then we can formulate the elastic wave propagation problem

$$
E u_{x x}-\rho u_{t t}=u_{t t} M \delta(x-l)
$$

with boundary conditions

$$
\begin{aligned}
\left.u_{x}\right|_{x=0} & =\frac{P(t)}{E} \\
\left.u_{x}\right|_{x-L} & =0
\end{aligned}
$$

where $E$ is the elastic module and $\rho$ is the density of material.

Let us divide beam into two domains (in front of the inclusion and beyond it). Then in both domains we will have the problem without inclusion and only in the point $x=l$ we will have the condition of conjunction

$$
\left[u_{x}\right]_{l}=\frac{M}{E} u_{t t}(l)
$$


Solving both problems by the method of Laplace transform we will have for Laplace transform of displacements in domains $\hat{u}^{(1)}(x, p), \tilde{u}^{(2)}(x, p)$

$$
\begin{aligned}
\tilde{u}_{x x}^{(1)}-\frac{p^{2}}{c^{2}} \tilde{u}^{(1)} & =0 \\
\tilde{u}_{x}^{(1)}(0) & =\frac{\tilde{P}}{E} \\
\tilde{u}_{x x}^{(2)}-\frac{p^{2}}{c^{2}} \tilde{u}^{(2)} & =0 \\
\tilde{u}_{x}^{(2)}(L) & =0
\end{aligned}
$$

with conjunction condition

$$
\begin{aligned}
\tilde{u}^{(1)}(l) & =\tilde{u}^{(2)}(l) \\
\tilde{u}_{x}^{(2)}(l)-\tilde{u}_{x}^{(1)}(l) & =\frac{M p^{2}}{E} \tilde{u}_{l}
\end{aligned}
$$

The solution of the problem (2.4) - (2.6) was found in the form

$$
\begin{array}{r}
\tilde{u}^{(1)}(x, p)=\frac{c \tilde{P}}{p}\left[\sinh \frac{p x}{c}-\tanh \frac{p l}{c} \cosh \frac{p x}{c}\right]+\tilde{u}_{l} \frac{\cosh \frac{p x}{c}}{\cosh \frac{p l}{c}} \\
\tilde{u}^{(2)}(x, p)=\tilde{u}_{l} \frac{\cosh \frac{p(L-x)}{c}}{\cosh \frac{p(L-l)}{c}}
\end{array}
$$

where

$$
\tilde{u}_{l}=-\frac{c \tilde{P}}{p} \frac{1}{\cosh \frac{p l}{c}} \frac{1}{M p c+\frac{\sinh \frac{p L}{c}}{\cosh \frac{p(L-l)}{c} \cosh \frac{p l}{c}}}
$$

The next problem is a problem of inverse Laplace transformation of functions $\tilde{u}^{(1)}, \tilde{u}^{(2)}$ or stress functions $\tilde{\sigma}^{(1)}=E \tilde{u}_{x}^{(1)}$ and $\tilde{\sigma}^{(2)}=E \tilde{u}_{x}^{(2)}$ These depend on the form of applied impulse $P(t)$ and on the value of inclusion mass and could be found in some particular cases. We must take into account the influence of the inclusion in the split problems. Since split takes place near the free end of the beam we will consider the propagation of the stress wave in the domain beyond the inclusion $(l<x<L)$. In this domain

$$
\tilde{\sigma}^{(2)}=\tilde{g}_{l} \sinh \frac{p(L-x)}{c}
$$

where $\tilde{g}_{l}$ can be represented in the form

$$
\tilde{g}_{l}=\frac{2 \tilde{P} e^{-p L / c}}{1+\delta p}\left[1+\frac{\delta p}{1+\delta p}\left(e^{-2 p l / c}+e^{-2 p(L-l) / c}\right)-\frac{1-\delta p}{1+\delta p} e^{-2 p L / c}\right]
$$

and $\delta=2 E / M c$ This expression we can simplify if inequality

$$
\delta p\left(e^{-2 p l / c}+e^{-2 p(L-l) / c}\right)-(1-\delta p) e^{-2 p l / c} \ll(1+\delta p)
$$


If (2.11) is fulfilled we can simplify (2.10) and then inverse Laplace transformation of $\tilde{\sigma}^{(2)}$ has the following form

$$
\sigma^{(2)}(x, t)=q(t-x / c)-q(t-(2 L-x) / c)
$$

where

$$
\tilde{q}(p)=\frac{\tilde{P}}{1+\delta p}
$$

It is easy to find the inverse transformation of $\tilde{q}$

$$
q(t)=\delta \int_{0}^{t} P(t-\tau) e^{-\delta t} d \tau
$$

Suppose $P(t)=P_{0} \exp -\theta t$ then

$$
\sigma(x, t)=\frac{\delta P_{0}}{\theta-\delta}\left[\left(e^{-\theta(t-x / c)}-e^{-\theta\left(t-\frac{2 L-x}{c}\right)}\right)-\left(e^{-\delta(t-x / c)}-e^{-\delta\left(t-\frac{2 L-x}{c}\right)}\right)\right]
$$

It is easy to show that in the beam without inclusion under the same loading

$$
\sigma(x, t)=P_{0}\left[e^{-\theta(t-x / c)}-e^{-\theta\left(t-\frac{2 L-x}{c}\right)}\right]
$$

Assumption expressed by (2.11) alter true stress field. However influence of the inclusion on the stress wave propagation is obvious and can be estimated qualitatively. So we can see that stress is decreased in corresponding points. It may be connected with partial wave reflection from the inclusion in the first domain.

\section{References}

[1] Panin V.E.,Lihachev V.A.and Grinjaev Yu.V.Structural levels of the deformation in solids (Nauka,Novosibirsk,1985)

[2] Koneva N.A.and Kozlov E.V.,Physica,Tomsk Univ.Etd.33(1990) 89-106.

[3] Prockuratova E.I.and Gaikov A.L.,Journal de Physic IV 1 (1991) 943-947.

[4] Prockuratova E.I.and Indeitchev D.A.,Dymat Journal 2(1995) 229-233.

[5] Prockuratova E.I.and Rogozhin V.V.,J.Mech.Beh.Mat. 5(1994) 417-428. 